\title{
Increasing genomic literacy among adolescents
}

\author{
Maya Sabatello, LLB, PhD ${ }^{1}$, Ying Chen, MD, MS, MA², Saskia C. Sanderson, PhD, CPsychol ${ }^{3,4}$, \\ Wendy K. Chung, MD, $\mathrm{PhD}^{5,6}$ and Paul S. Appelbaum, $\mathrm{MD}^{1}$
}

Purpose: Adolescents increasingly need to be "genomics literate," and may engage more with video educational formats than traditional written formats. We conducted a pilot study to assess and compare the impact of two modes of education about genome sequencing (GS) on adolescents' genomic knowledge and genomicrelated decisions.

Methods: Using an online survey, 43 adolescents ages 14-17 years were randomly assigned to watch a video or read a pamphlet about GS. Measures included pre- and postintervention assessment of genomic knowledge, perceived utility of these materials for decisions about participating in genetic research, interest in receiving GS results, and overall satisfaction with these materials. Analyses described results for all participants and compared results between intervention groups.

Results: Self-reported genomic knowledge increased overall $(p<0.001)$. Postintervention knowledge about GS limitations was higher among video group than pamphlet group participants $(p=0.038)$. More video group than pamphlet group participants expressed satisfaction with the material's understandability ( $45 \%$ vs. $29 \%)$ and suitability (91\% vs. $76 \%$ ). Interest in receiving personal GS results was significantly associated with being female $(p=0.01)$ and younger (14-15 years vs. $16-17$ years) $(p=0.002)$.

Conclusion: A video format may be preferable for increasing genomic literacy among adolescents. Further research with adolescents is needed to better understand how gender and age may impact genomic decisions and preferences.

Genetics in Medicine (2019) 21:994-1000; https://doi.org/10.1038/s41436018-0275-2

Keywords: genome sequencing; genomic literacy; adolescent education

\section{INTRODUCTION}

Genomic literacy among children and young people, especially adolescents, is of increasing importance. The ever-expanding prevalence of genetics in everyday life includes ancestry testing; the increasing number of clinical genetic tests; the rise of research endeavors that utilize genome and exome sequencing, enroll pediatric participants, and offer families genetic results; and the professional opportunities in genomics that increasingly are available for adolescents as they train to enter the workforce (e.g., bioinformatics, genetic counseling). All of these point to the need to develop genomic educational material for adolescents. Such educational material can help adolescents to think about whether to participate in genomic research; to understand the risks and benefits involved in such participation, including return of genetic results; ${ }^{1}$ to improve their knowledge of the limitations of genetic testing or sequencing and the ability to predict disease risk; and to begin forming their attitudes and beliefs about genomic medicine and research. Such genomic knowledge among adolescents also may be helpful as adolescents support older family members who may not understand genomic information and seek their opinions.

However, current genomic educational resources and curricula in high schools fail to address this need adequately. ${ }^{2}$ Studies indicate that high school biology textbooks are deficient in their explanations of key issues, such as gene-environment interactions, ${ }^{3}$ and that only $28 \%$ of high school students taking the ACT receive a score indicating college readiness for biology, including key concepts in evolution and genetics. ${ }^{4}$ A study of 1600 teachers (including 1471 from the United States; $65 \%$ of them high school teachers) further found that although most of them taught life science courses, only $25 \%$ reported teaching contemporary topics in genomics, such as genomic DNA sequencing, and many $(\sim 45 \%)$ felt that they lacked the expertise and teaching resources to do so. ${ }^{2}$ A study that assessed the quality of genetics education in the United States for grades 9-12 concluded that the average standard was poor, with more than $85 \%$ of states receiving overall scores of Inadequate. ${ }^{5}$ Following the 19 benchmark concepts of the American Society of Human Genetics, the study found that only 5 core

\footnotetext{
${ }^{1}$ Center for Research on Ethical, Legal \& Social Implications of Psychiatric, Neurologic \& Behavioral Genetics, Department of Psychiatry, Columbia University, New York, NY, USA; ${ }^{2}$ Division of Biostatistics, New York State Psychiatric Institute, New York, NY, USA; ${ }^{3}$ Department of Behavioural Science and Health, University College London, London, UK; ${ }^{4}$ Department of Clinical Genetics, Great Ormond Street Hospital, London, UK; ${ }^{5}$ Department of Pediatrics, Columbia University, New York, NY, USA; ${ }^{6}$ Department of Medicine, Columbia University, New York, NY, USA. Correspondence: Maya Sabatello (ms4075@columbia.edu)
} 
concepts, relating to the biological nature of DNA, single-gene Mendelian pattern of transmission, and evolution, were adequately addressed in high school curricula. The other 14 core concepts about polygenic patterns of inheritance, gene expression and regulation, and genetic variation were found to be either absent or inadequately addressed. These deficiencies are significant because adolescents may not learn about key concepts that not only reflect contemporary genomic knowledge practically but also impact their health and life-planning decisions.

Closing these educational gaps is difficult, given that statemandated curricula do not include many contemporary genomics concepts, ${ }^{2}$ but it can be facilitated by developing educational materials that can be widely and freely disseminated and that high school students can explore independently or that teachers can easily incorporate into classroom discussions. However, there is little research on which format for presenting genomic information is most effective in educating adolescents. A common presumption in science education is that visualizations can help clarify abstract or complex concepts and that the use of nonwritten alternate modalities can better engage students and improve their understanding of the material. ${ }^{6}$ The scalability of videos makes them particularly attractive due to their potential for rapid and extensive dissemination. These presumptions may be more relevant for adolescents, who regularly use the Internet, social media, and apps and who frequently have smartphones. However, the effectiveness of educational videos about genomics that are publicly available on the Internet is largely unknown.

One exception is a study ${ }^{7}$ in which an animated educational video was developed and compared with an information pamphlet about genome sequencing (GS) in an experimental online survey of adults ( $>18$ years old) assessing their comparable accessibility and effectiveness. The study found significant differences in knowledge between the video group and no-information group, and importantly, the video was more accessible and effective than written information for people across educational, racial, and ethnic backgrounds. The study also found that video group participants demonstrated a significant increase from preintervention to postintervention in knowledge of lifestyle genomics (i.e., how a person's health habits, such as diet and exercise, can affect genetic predispositions to diseases) and of the benefits-but not limitations-of GS. However, it is unknown whether the video would yield similar results among adolescents.

In the present paper, we report on findings from a pilot study that was conducted with adolescents (ages 14-17). The study aimed to assess and compare the impact of two modes of education about GS (a video versus a pamphlet) on adolescents' genomic knowledge, perceived utility of these materials for decisions to participate in genetic research, interest in receiving personal GS results, and overall satisfaction with these approaches. Our study did not include a group that received no education because of the evidence reviewed above about the deficiencies in genetic education in high schools, ${ }^{2}$ and it would have been hard (and possibly discouraging) for the participants to answer the subsequent questions about GS without being provided with information about it. ${ }^{8}$

\section{MATERIALS AND METHODS}

\section{Participants}

An anonymous, approximately 20-minute, online survey was administered to 43 adolescents aged 14 to 17 . Participants were recruited by Touchstone Research, Inc. (TSR), a professional research firm that has assembled an online, double opt-in, Internet-based Kids and Family panel (http:// touchstoneresearch.com/youth-families/). Both parental consent and adolescents' assent were obtained after they were provided with information about the study, including a simple-language explanation of key genetic terms ("genetics," "genetic testing," and "genomic research"). Parents were asked to indicate their demographic information (age, sex, education, employment, household income) and their child's sex, age, and race/ethnicity.

\section{Study design}

The survey began with a baseline assessment of participants' genomic knowledge. Participants were then randomly assigned to one of two modes of education about GS (video or pamphlet (termed "handout" in the survey); see further information below) and invited to answer further questions after confirming that they had watched/read the material. TSR administered the survey and offered participants the equivalent of $\$ 10$ for their participation. The researchers did not have access to participants' identifying information, and they were blinded as to the participants' assignment to the intervention groups. The institutional review board (IRB) at the New York State Psychiatric Institute approved the study.

The educational material that participants received was either the 11-minute animated video "Whole Genome Sequencing and You" (https://www.youtube.com/watch? $\mathrm{v}=\mathrm{IXamRS85hXU)}$ or a revised, child-friendly version of an informational pamphlet, both developed in previous research. ${ }^{7}$ To ensure that the study was adolescent-friendly, the reading levels of the invitation letter, survey, and informational pamphlet were assessed using Word's Flesch-Kincaid Grade Level test, and adjusted to accommodate seventh and eighth graders (adolescents 13-14 years old). The revised pamphlet comprised 1,063 words and was estimated to require 6-8 minutes to read (see Supplemental File S1). Additionally, three educators with substantial experience in teaching adolescents ages 14-17 reviewed the material and provided feedback on the content and its understandability.

\section{Measures}

The survey included the questions and measures used by Sanderson et al.'s 2015 study to evaluate participants' pre- and postintervention genomic knowledge, perceived utility of the material, interest in participating in genetic research and 
receiving results, and overall satisfaction with their respective educational material. ${ }^{7}$

\section{Knowledge}

The survey included measures to assess self-rated and objective knowledge. Self-rated knowledge was assessed using seven key terms: whole-genome sequencing, genome, gene, DNA, chromosome, pharmacogenetics, and DNA variant of unknown significance (for each term, participants marked whether they "know the meaning of," "are aware of," or "never heard of" the term). ${ }^{9}$ Summary of scores pre- and postintervention were coded, with lower score indicating more knowledge.

Objective knowledge was assessed using 11 items: 5 statements about benefits of sequencing (e.g., "Genome sequencing may give a person information about their chances of developing several different diseases"), 5 about limitations of sequencing (e.g., "Once a variant in a gene that affects a person's risk of a disease is found, that disease can always be prevented or cured"), and 1 item on "lifestyle genomics" ("A person health's health habits, such as diet and exercise, can affect whether or not their genes cause diseases"). ${ }^{10}$ The response options for each statement were on a 1-5 Likert scale ( $1=$ "strongly agree" and $5=$ "strongly disagree"), and were recoded in the analysis into three groups: for each true statement, "strongly agree" was coded as 2 , "somewhat agree" was coded as 1 , and the other three possible responses were coded as 0 . False statements were reversed coded and then grouped into the same categories. Summary of mean scores pre- and postintervention were coded, with lower scores indicating more accurate answers. The item on "lifestyle genomics" was recoded, with 5 corresponding to high knowledge, and 1 to low knowledge.

\section{Perceived utility}

Perceived utility of the information to support decisionmaking about participating in GS research was assessed using one item ("Would you have found this (video/handout) helpful if you were deciding whether to participate in a study utilizing whole-genome sequencing?"). ${ }^{11}$ Participants were also asked "Would you want to participate in this genetic research study?" and "Would you want to receive your personal whole-genome sequencing results?"12 The response options for both questions were on a 1-4 Likert scale ( $1=$ "no, definitely not" and $4=$ "yes, definitely yes"; the question on receiving personal results also included $5=$ "don't know" and $6=$ "it would depend").

\section{Satisfaction}

Participants' satisfaction with the video or pamphlet was assessed using two questions about the amount and understandability of the material and the mean score of 7 items about the material's design and organization that comprised an "individual satisfaction scale" ( $1-5$ scale; $1=$ very satisfied/ strongly agree, $5=$ very dissatisfied/strongly disagree). ${ }^{13}$ Cronbach's $\alpha$ was 0.73 , indicating acceptable reliability.

\section{Statistical analyses}

Study data were analyzed using IBM Statistics SPSS 24.0. Descriptive statistics were used for demographic characteristics. Paired sample $t$ test was used to assess the differences between means of single pre- and postquestionnaires. Reliability of variables combined to create the satisfaction scale score was tested with Cronbach's $\alpha$. The differences preand postintervention were tested with nonparametric statistics (Kolmogorov-Smirnoff tests). Between-group differences were compared with Pearson $\chi^{2}$ tests. Generalized linear models (GLMs) were used to test the impact of demographic characteristics on outcome variables. Repeated measures analysis of variance (ANOVA) was used to test pre- and postintervention self-rated knowledge differences between intervention groups and among age groups. All tests are twosided and a $p$ value $<0.05$ was considered significant.

\section{Demographic characteristics}

\section{RESULTS}

Forty-three adolescents ages 14-17 completed the survey, of whom $22(51.2 \%)$ were female; mean age was 15.3 years $(\mathrm{SD}=1.16)$. Twelve participants identified as Hispanic, 33 as white, 7 as African American, 3 as Asian, 2 as American Indian or Alaska Native, and 1 as Native Hawaiian or other Pacific Islander (these numbers include 3 participants who self-identified with more than one race). Of the participants, $46.5 \%$ had a household annual income $<\$ 74,999$ (as per the 2016 census, the mean income in the United States is $\$ 73,298$ ) (Table 1). Twenty-two participants viewed the video and 21 read the pamphlet.

\section{Knowledge}

The proportion of participants responding that they "never heard of the term" was lower postintervention compared with preintervention for whole-genome sequencing, genome, pharmacogenomics, and DNA variant of unknown significance. The increase in self-rated knowledge was observable especially for the latter two terms: 11/20 (55\%) and 9/23 (39\%) participants changed their response for each from "never heard of the term" preintervention to "know the meaning of the term" postintervention. However, there were no differences between video and pamphlet groups. In addition, no changes were found in self-rated knowledge of the other terms (many participants already knew the meaning or had heard of the terms gene and DNA before the intervention and only two participants responded that they never heard of the term chromosome). Overall self-rated knowledge increased from $11.54(\mathrm{SD}=5.05)$ preintervention to $16.25(\mathrm{SD}=5.03)$ postintervention $(t=5.06, \mathrm{df}=38$, $p<0.001)$. However, no differences were found between age groups $(\mathrm{F}=1.556, \mathrm{df}=3, p=0.217)$, or when adjusting for intervention groups $(\mathrm{F}=1.703, \mathrm{df}=3, p=0.185)$.

Overall objective knowledge (including benefits, limitations, and lifestyle) also increased in the overall sample from 11.7 $(\mathrm{SD}=5.52)$ preintervention to $14.7(\mathrm{SD}=4.62)$ postintervention. Again, there was no difference between the video and 
Table 1 Sociodemographic characteristics

Total $(N=43)$

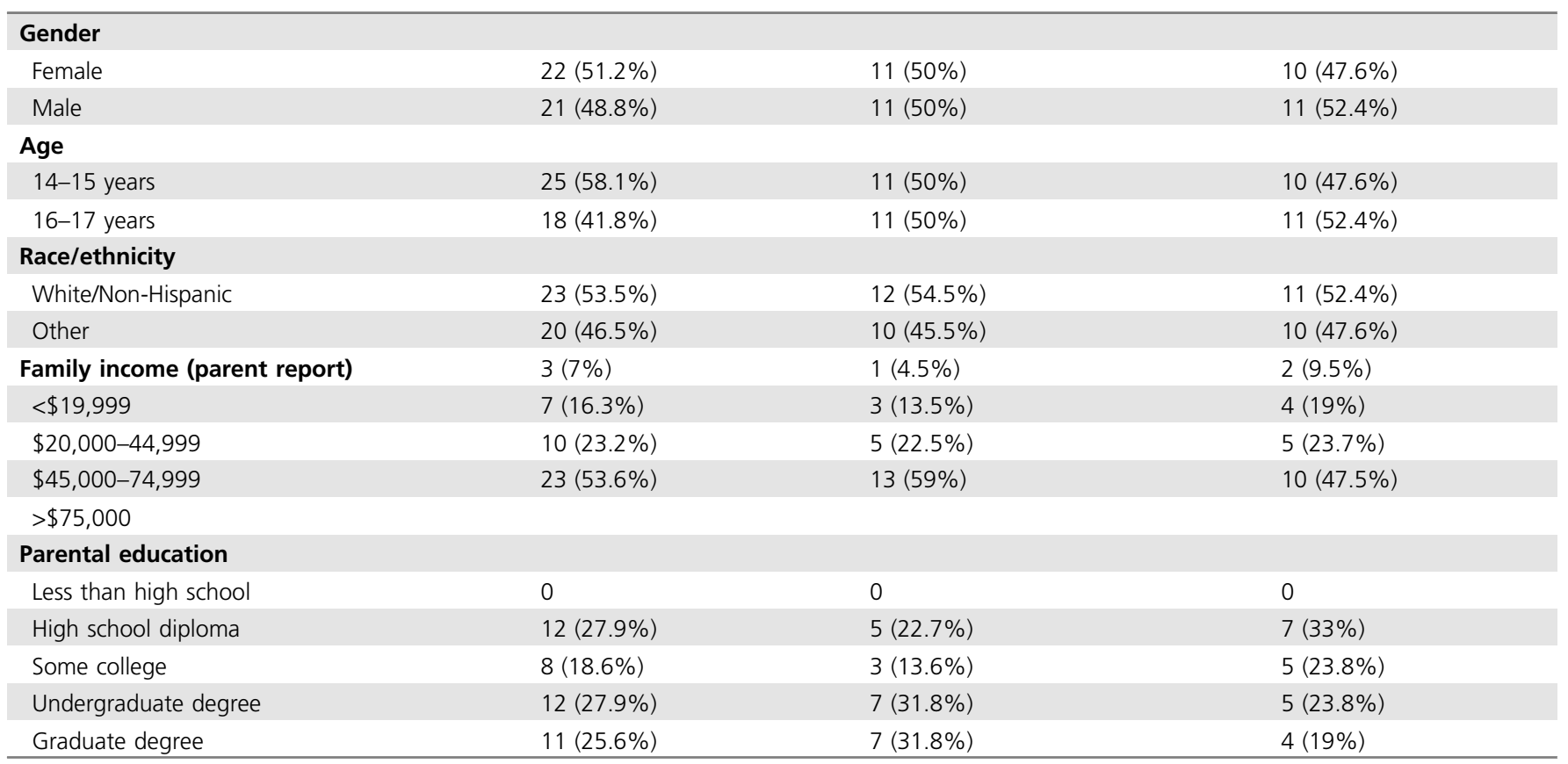

Table 2 Objective knowledge compared between experimental groups Objectively assessed knowledge

\begin{tabular}{|c|c|c|c|c|c|c|}
\hline & \multirow{2}{*}{\multicolumn{2}{|c|}{ Written information group $(n=21)$}} & \multirow{3}{*}{ Total $n=43$} & \\
\hline & \multicolumn{2}{|c|}{ Video information group $(n=22)$} & & & & \\
\hline & Pre & Post & Pre & Post & & \\
\hline $\begin{array}{l}\text { Knowledge about } \\
\text { benefits (0-10 scale) }\end{array}$ & 5.7 & 7.5 & 4.3 & 6.6 & & \\
\hline Mean (SD) & 2.9 & 2.1 & 2.8 & 2.6 & & \\
\hline & & & & & $F(1)=0.71 ; p=0.40$ & $p=0.30$ \\
\hline Mean (SD) & 3.1 & 2.6 & 2.7 & 2.8 & & \\
\hline & & & & & $F(1)=2.17 ; p=0.15$ & $p=0.04$ \\
\hline $\begin{array}{l}\text { Knowledge about } \\
\text { lifestyle ( } 1-5 \text { item) }\end{array}$ & 3.5 & 4.2 & 3.4 & 4.3 & & \\
\hline Mean (SD) & 1.1 & 1.0 & 1.3 & 0.8 & $\mathrm{~F}(1)=0.14 ; p=0.71$ & $p=0.52$ \\
\hline
\end{tabular}

pamphlet intervention groups $(p=0.895)$ (Table 2$)$ or when adjusting for age groups $(\mathrm{F}=0.574, \mathrm{df}=3, p=0.636)$. However, postintervention knowledge about limitations of genomic sequencing was significantly higher among video group than pamphlet group participants $(p=0.038)$ (Figs. 1-3).

\section{Perceived utility}

Almost all of the participants in both the video and pamphlet groups reported that the educational material they received would be helpful if they were deciding whether to participate in a GS study (20 participants from each intervention group responded "yes"). There was no difference between the two intervention groups $\left(\chi^{2}=0.46\right.$, $\mathrm{df}=1, p=0.58)$.

\section{Research participation and return of results}

The majority of participants expressed interest in participating in genetic research and receiving personal GS results. Overall, $77 \%$ of participants answered "yes, definitely" or "yes, probably" to research participation, and $88 \%$ responded "yes, definitely" or "yes, probably" to receiving results. 


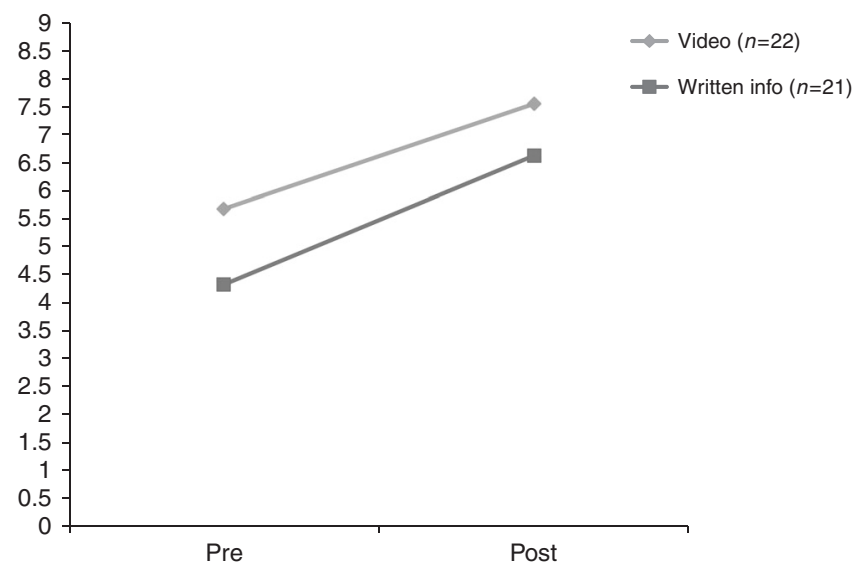

Fig. 1 Knowledge about benefits

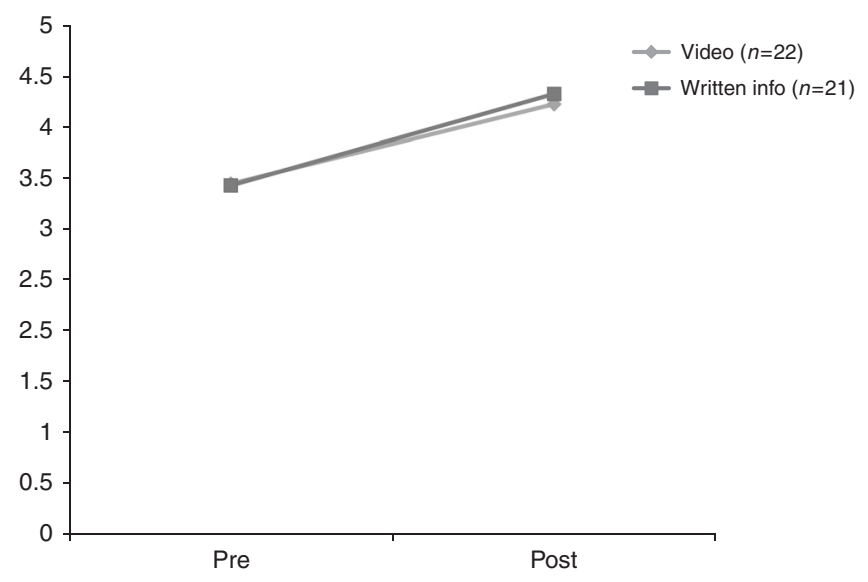

Fig. 2 Knowledge about lifestyle

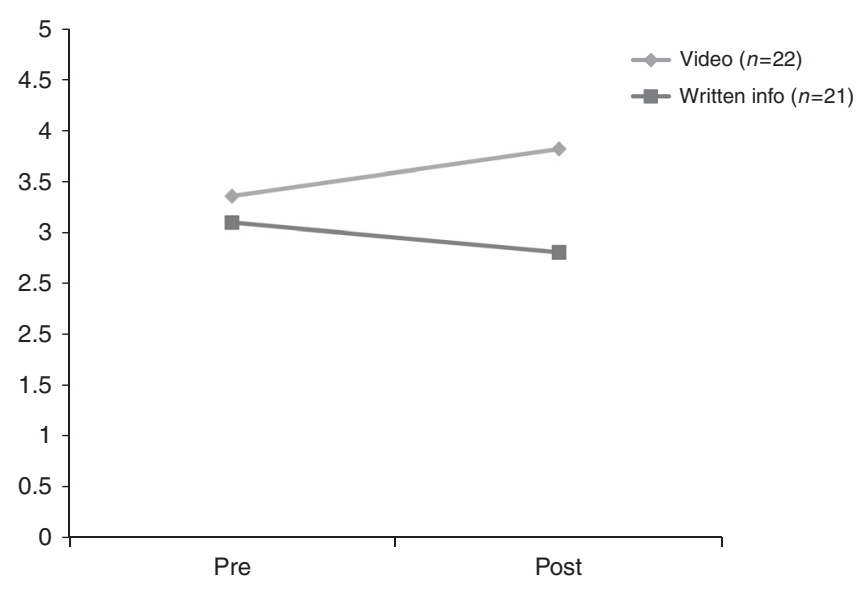

Fig. 3 Knowledge about limitations

These differences in interest in participating in genetic research and the decision whether to receive GS results were not statistically significant by intervention group (respectively, Pearson $\chi^{2}=3.795, p=0.285, \mathrm{df}=3$; Pearson $\chi^{2}=5.125, \mathrm{df}$ $=3, p=0.163)$. However, adolescent females were more likely than males to express interest in receiving results $(p=0.004)$.
To further explore whether participants' interest in receiving personal results from genetic research was influenced by demographic factors, responses to the interest question were dichotomized into "yes, definitely" and "yes, probably" vs. "undecided"/“don't know" responses (no participant responded "no, definitely not" or "no, probably not"). Interest in receiving results was significantly associated with younger age $(p=0.02)$ and $\operatorname{sex}(p=0.01)$. Younger females (14 to 15 years old) expressed greater preferences to receive results.

\section{Satisfaction}

Overall satisfaction with the educational materials was high. The mean satisfaction scale score was $1.45(\mathrm{SD}=0.49)$ for the video group and $1.51(\mathrm{SD}=0.34)$ for the pamphlet group (possible range of 1 to 5 , where lower scores represent higher satisfaction). There was no statistically significant difference in the satisfaction scores between the video and pamphlet groups $(Z=0.87, \mathrm{df}=41, p=0.43)$. However, $45 \%$ of the video group said their educational material was "very easy" to understand compared with $29 \%$ of the pamphlet group participants $\left(\chi^{2}=3.02, \mathrm{df}=2, p=0.22\right)$ and $91 \%$ of the video group said they found the amount of information in the educational material to be "the right amount" compared with $76 \%$ of the pamphlet group participants $\left(\chi^{2}=2.09, \mathrm{df}=2\right.$, $p=0.35$ ).

\section{DISCUSSION}

In this pilot study, we explored how adolescents responded to GS educational material that had previously been shown to improve adults' understanding of GS. We explored whether the materials would have a similar impact among adolescents, and also whether the animated video was preferable to an adolescent-friendly written pamphlet in terms of adolescents' satisfaction with the material and its usefulness in improving their genomic knowledge. Because our sample size was limited, our findings are preliminary. Nonetheless, they provide initial insight into adolescents' views on genomic research, return of genomic results, and preferred formats for learning about genomics among this understudied but important population.

We found that, similar to the previous research with adults, ${ }^{7}$ adolescent participants' self-rated and objective genomic knowledge significantly increased following both educational interventions, though, unlike in the study by Sanderson et al., the video format was more effective than the pamphlet in increasing knowledge of the limitations of sequencing. The increase in self-rated knowledge was especially notable for key GS-relevant terms that many participants had not heard of before the intervention (e.g., "whole-genome sequencing"). This finding corresponds with the results of studies about the genomic educational gap among high school students and is reassuring about the prospect of increasing genomic literacy among adolescents. It is further interesting that while no differences in objective genomic knowledge were found between adolescent age groups, the 
adolescents in our study appeared to have higher objective genomic knowledge than adults in previous research. For example, in a previous study, ${ }^{7}$ adults' mean baseline knowledge scores about the benefits of GS were $3.28,3.55$, and 3.71 in each of three experimental groups, whereas in the present study adolescents' mean baseline scores on the same scale were 4.3 and 5.7 in the two experimental groups. Although this difference could be due to other unmeasured differences between the two studies, it is consistent with previous data suggesting that adolescents' knowledge of genetics is at least as good as adults, ${ }^{14}$ and perhaps reflects increasing genetics and genomics education in schools. ${ }^{2}$ Future research would benefit from further exploring the differences between adolescents and adults' understanding about GS, as this might inform age-stratified educational strategies.

Although participants' satisfaction and perceived utility were high for both educational formats, our results support previous findings with adults that the video was more accessible and easier to understand than the pamphlet. ${ }^{7}$ Additionally, our study found that adolescents who were exposed to GS educational material expressed strong interest both in participation in genetic research and in receiving research results. We also found that age and gender were significantly associated with interest in receiving genomic results, with younger, female adolescents expressing the most interest.

Our findings have potentially important-albeit preliminary-implications for efforts to increase genomic literacy among adolescents. The increased genomic knowledge postintervention highlights the usefulness of easy-tounderstand GS educational material for improving genomic literacy. Although both educational formats achieved this goal, participants' higher satisfaction with the video suggests that a dynamic medium may be a preferable way to convey genomic messages to adolescents.

The association between participants' gender and their interest in receiving personalized genomic results further raises important issues for consideration. This finding is consistent with-and expands on-results from studies on single-gene testing, suggesting that women are more likely to undergo predictive genetic testing ${ }^{15,16}$ and that $15-17$-yearold females are significantly more willing than males to be tested for Tay-Sachs and hypercholesterolemia. ${ }^{17}$ Although our sample size precludes definitive conclusions, this finding on gender differences suggests that the genderization of genomic knowledge may develop by adolescence. That is, women's sense that they carry the lion's share of genomic responsibility for themselves, their offspring, and their extended family members, as expressed, e.g., by willingness to make behavioral changes, such as changing diet and increasing physical activity, and to have more regular medical monitoring in response to genetic information, ${ }^{15,18}$ may already be in place by that age. This may be because of greater curiosity or because genetic testing for familial breast cancer, and carrier screening more generally, has traditionally been more available to women. Further research is needed to understand the scope, reasons, and implications of this early onset of gendered genomic responsibility.

This pilot study has limitations, primarily the small sample size, which precludes more nuanced data analyses. Additionally, given the hypothetical nature of the survey, it is impossible to know whether adolescents' interest will translate into actual enrollment in genomic studies and increased uptake of results. A pilot program in Australia that offered high school students genetic susceptibility testing for hereditary hemochromatosis, an adult-onset, treatable, and preventable disorder that causes the body to absorb too much iron from the diet, showed high interest and uptake: a total of 5,757 students were subsequently tested for this condition. ${ }^{19}$ However, the uptake may be lower if testing is offered to individuals, rather than as a public health screening program, especially in the United States, where there is no national health-care system and there is fear about insurability if personal genetic information is disclosed. ${ }^{20}$ As studies with adults indicate, there is often a gap between participants' hypothetical interest in undergoing predictive genetic testing and the actualization of such interest, that is, between intention and behavior. ${ }^{16,21,22}$

Nonetheless, our findings suggest that adolescents' genomic literacy can increase when provided with adolescent-friendly educational material about GS, and that adolescents from the general public (rather than or in addition to the conventional focus on pediatric patients ${ }^{23}$ ) are capable of learning about genomic information. Moreover, our findings-and limitations-highlight the need for further exploration of adolescents' views on genomic testing and research. Indeed, the rise of genomic research, including the All of Us Research Program, which plans to enroll children of all ages, will likely make scenarios such as those described in our survey a reality for many adolescents. Better understanding of adolescents' preferences and perspectives about genomic research will help in developing tailored policies and improving translational efforts as this population transitions into genomic citizens.

\section{ELECTRONIC SUPPLEMENTARY MATERIAL}

The online version of this article (https://doi.org/10.1038/s41436018-0275-2) contains supplementary material, which is available to authorized users.

\section{ACKNOWLEDGEMENTS}

This work was supported by grant funding from the National Institute of Health (NIH), K01HG008653 and P50HG007257, the Columbia University Precision Medicine \& Society Program, and the Once Upon a Time Foundation. We thank Cassius Gil, Josh Goren, and Scot Nourok for their comments and insights as educators on the study material.

\section{DISCLOSURE}

The authors declare no conflicts of interest. 


\section{REFERENCES}

1. Sabatello M, Appelbaum PS. Raising genomic citizens: adolescents and the return of secondary genomic findings. J Law Med Ethics. 2016;44:292-308.

2. National Human Genome Research Institute. Genomic Literacy Education and Engagement (GLEE) white paper: K-16 Working Group. 2017. https://www.genome.gov/pages/about/od/ecib/glee/glee_white_paper_ k-16_wg.pdf.

3. Dougherty MJ. Closing the gap: inverting the genetics curriculum to ensure an informed public. Am J Hum Genet. 2009;85:6-12.

4. Battelle B, Biotechnology Institute. Departments of Pediatrics and Medicine. Taking the pulse of bioscience education in America: a state by state analysis. 2009. https://pages.wustl.edu/files/pages/imce/ scienceandtechnology/bio_09_summary_report.pdf.

5. Dougherty MJ, Pleasants C, Solow L, Wong A, Zhang H. A comprehensive analysis of high school genetics standards: are states keeping pace with modern genetics? CBE Life Sci Educ. 2011;10:318-327.

6. Annetta L, Minogue J, Holmes SY, Cheng M-T. Investigating the impact of video games on high school students' engagement and learning about genetics. Comput Educ. 2009;53:74-85.

7. Sanderson SC, Suckiel SA, Zweig M, Bottinger EP, Jabs EW, Richardson LD. Development and preliminary evaluation of an online educational video about whole-genome sequencing for research participants, patients, and the general public. Genet Med. 2016;18:501-512.

8. Kung JT, Gelbart ME. Getting a head start: the importance of personal genetics education in high schools. Yale J Biol Med. 2012;85:87-92.

9. Ishiyama I, Nagai A, Muto K, Tamakoshi A, Kokado M, Mimura K, et al. Relationship between public attitudes toward genomic studies related to medicine and their level of genomic literacy in Japan. Am J Med Genet A. 2008;146a:1696-1706.

10. Kaphingst KA, Facio FM, Cheng MR, Brooks S, Eidem H, Linn A, et al. Effects of informed consent for individual genome sequencing on relevant knowledge. Clin Genet. 2012;82:408-415.

11. O'Connor A, Cranney A. User manual-acceptability. 2002. https:// decisionaid.ohri.ca/docs/develop/User_Manuals/UM_Acceptability.pdf.

12. Sanderson SC, Diefenbach MA, Zinberg $R$, Horowitz $C R$, Smirnoff $M$, Zweig $\mathrm{M}$, et al. Willingness to participate in genomics research and desire for personal results among underrepresented minority patients: a structured interview study. J Community Genet. 2013;4:469-482.
13. Andermann AA, Austoker J, Watson EK, Lucassen AM, Mackay J. Development and evaluation of a general information leaflet for women with a family history of breast cancer. J Cancer Educ. 2002;17:155-160.

14. Rew L, Mackert M, Bonevac D. Cool, but is it credible? Adolescents' and parents' approaches to genetic testing. West J Nurs Res. 2010;32: 610-627.

15. d'Agincourt-Canning L, Baird P. Genetic testing for hereditary cancers: the impact of gender on interest, uptake and ethical considerations. Crit Rev Oncol Hematol. 2006;58:114-123.

16. Forrest L, Delatycki M, Curnow L, Gen Couns M, Skene L, Aitken M. An audit of clinical service examining the uptake of genetic testing by at-risk family members. Genet Med. 2012;14:122-128.

17. Harel A, Abuelo D, Kazura A. Adolescents and genetic testing: what do they think about it? J Adolesc Health. 2003;33:489-494.

18. Godino JG, van Sluijs EM, Marteau TM, Sutton S, Sharp SJ, Griffin SJ. Lifestyle advice combined with personalized estimates of genetic or phenotypic risk of type 2 diabetes, and objectively measured physical activity: a randomized controlled trial. PLoS Med. 2016;13: e1002185.

19. Delatycki MB, Wolthuizen M, Collins V, Varley E, Craven J, Allen KJ, et al. Implementation of ironXS: a study of the acceptability and feasibility of genetic screening for hereditary hemochromatosis in high schools. Clin Genet. 2010;77:241-248.

20. Armstrong K, Putt M, Halbert CH, Grande D, Schwartz JS, Liao K, et al. The influence of health care policies and health care system distrust on willingness to undergo genetic testing. Med Care. 2012;50:381-387.

21. Sanderson SC, O'Neill SC, Bastian LA, Bepler G, McBride CM. What can interest tell us about uptake of genetic testing? Intention and behavior amongst smokers related to patients with lung cancer. Public Health Genomics. 2010;13:116-124.

22. Ropka ME, Wenzel J, Phillips EK, Siadaty M, Philbrick JT. Uptake rates for breast cancer genetic testing: a systematic review. Cancer Epidemiol Biomarkers Prev. 2006;15:840-855.

23. Levenseller BL, Soucier DJ, Miller VA, Harris D, Conway L, Bernhardt BA. Stakeholders' opinions on the implementation of pediatric whole exome sequencing: implications for informed consent. J Genet Couns. 2014;23:552-565. 